\title{
Discovery Driven Innovation
}

\author{
August 22, 2019 \\ Financial Services Development and Cybersecurity Pioneering \\ https://cyberdevfinsec.blogspot.com/
}

\section{Introduction}

Many entities strive to be innovative in their pursuits, however, history has shown that some of the most innovative advances have occurred when the particular efforts are focused on something else. Some of the greatest innovations have happened by chance, or by an error in regular procedure or experimentation, or even a byproduct of an alternate use of existing technology. For this post, we'll look at innovations brought on by Serendipity, Errors, or Exaptation.

\section{Serendipity}

Napier and Hoang Vuong (2013) define serendipity as "the ability to recognize and leverage or create value from unexpected information." The interesting part about serendipity is that it the innovation may have been sitting in plain sight and has been waiting for discovery for ages. The mere instance of someone making the connection that the phenomenon could be used otherwise, tends to make this unpredictable in nature. Fink \& Reeves \& Palma \& Farr (2017) note that for every opportunity for serendipity there is the potential for an instance of an antiserendipitous event. Such assertions might make one think there is a universal balance in the universe that for every positive serendipitous event in existence, there is a negative event balancing out the equation. Crampton (2019) notes that some scientists design their experiments in a way to increase the chances of a serendipitous event.

One serendipitous discovery made its way into virtually every kitchen in the modern world. Percy Spencer was conducting experiments with a microwave-emitting magnetron that was often used in the internal mechanism of radar arrays. As he was experimenting with the device, he noticed that a chocolate bar in his pocket began to melt. That moment lead to the evolution of a new and effective game-changer in the culinary world. To this day, college students alike owe this discovery kudos for making the late-night study snack a reality.

\section{Errors Resulting in Discovery}


Innovation can at times be the result of an error in judgement or procedure. A chemist may accidently introduce the wrong volume of reactants and wind up with a new non-organic formula for moldable plastic. Likewise, a mistake in medical protocol may lead to a new technique to treating a medical diagnosis. Professional and academic communities often make discoveries from analyzing errors made by other colleges and find ways to turn the errors into a viable solution or discovery.

An example of discovery from a mistake or error is the discovery of artificial sweetener. Noted researcher Constantin Fahlberg was busy at work in his Johns Hopkins University lab. Upon taking a break, he neglected to wash his hands. An error in judgement for any seasoned lab worker, one that could lead to a quick case of death in some cases. While eating his meal, he noticed an oddly sweet flavor to his meal. Thus, an error in procedure led to the discovery of artificial sweetener. Fahlberg and Remsen, published their findings and developed a highly lucrative alternative to sugar.

\section{Exaptation}

Andriani and Cattani (2016) note that exaptation details a discontinuous evolutionary process that contains a short jump from one function to another. A biological example would be the evolution of feathers. Nature first designed feathers as a means for keeping in heat and regulating body temperature in birds. Over time, the use of feathers then shifted to facilitating the act of flight. The important thing to remember about exaptation is that it doesn't follow an expected evolutionary path. The innovation jumps from one function to a completely different function.

One such innovation as a result of exaptation can be illustrated by Corning Inc. transitioning from glass blowing to fiber optic cabling. Corning was known in the market for producing quality glass products. Advances in technology allowed for data transmissions to move faster and more accurately by using glass as a medium to transmit data using pulses of light. With Corning having expertise in glass construction, the opportunity arose to take this knowledge and develop new methods to creating the glass-based wiring to revolutionize telecommunications infrastructure.

\section{Summary}

To sum it up, not all innovations are planned or come from a clear evolutionary path. Phenomenon that are hiding in plan sight for centuries often come to light when someone takes notice of the inherent opportunity that is available. Likewise, an error in regular procedures or engineering has the potential to change the course of an already established norm. Such errors make it possible to come up with new innovations that can alter life as we know it. Lastly, exaptation provides the opportunity for specific attributes to make a functional 
jump from one course of evolution to a completely different course of service. A material that has not value at one particular time may later become a rare commodity that is priceless as a result of an exaptation jump.

\section{References}

Andriani, P.,Cattani, G. (2016). Exaptation as source of creativity, innovation, and diversity: introduction to the Special Section. Industrial and Corporate Change, 2016, Vol. 25, No. 1, 115-131. doi: 10.1093/icc/dtv053

Crampton, Linda (2019, March 15). Serendipity: The Role of Chance in Making Scientific Discoveries. Retrieved from https://owlcation.com/stem/Serendipity-The-Role-ofChance-in-Making-Scientific-Discoveries

M. A. Fink, T \& Reeves, M \& Palma, R \& S. Farr, R. (2017). Serendipity and strategy in rapid innovation. Nature Communications. 8. doi:10.1038/s41467-017-02042-w.

Napier, Nancy K. and Hoang Vuong, Quan. (2013). "Serendipity as a Strategic Advantage?". Strategic Management in the 21st Century, 1, 175-199. 